\title{
STATISTICS OF THREE-BODY EXPERIMENTS
}

\author{
M. J. VALTONEN
}

Institute of Astronomy, Cambridge, U.K.

\begin{abstract}
A large number of three-body interactions involving one initial binary have been studied by a numerical regularization technique. In each set of experiments some parameters have fixed values, whereas others are selected by uniform sampling of the corresponding distribution functions. Similar statistical results are obtained for different random number sequences at a lower integration accuracy.

This experimental approach permits an approximate determination of the final distributions of eccentricity, velocity and lifetime. These results show a strong dependence on the total angular momentum, total energy and the mass range, whereas other parameters are usually of secondary importance.
\end{abstract}

\section{Introduction}

Detailed numerical studies of the three-body problem reveal a considerable complexity of motions (e.g. Szebehely and Peters, 1967) which at first sight appear to limit the usefulness of a qualitative description of the dynamical behaviour. Thus each individual example looks different and the computed orbits are sensitive to numerical errors as well as to changes in the initial conditions. Nevertheless, characteristic trends begin to emerge when the results of many calculations are combined. Such investigations have mainly been concerned with planar motions in which the three particles are initially at rest (Agekyan and Anosova, 1967, 1968; Anosova, 1969a; Szebehely, 1972). The recent two-dimensional study by Standish (1972) consists of 800 examples, mostly with nonzero starting velocities which also enable the angular momentum dependence to be analysed.

In the present investigation we adopt the method of statistical sampling of initial conditions in three dimensions, the only restriction being that two of the particles form a binary initially. A total of 25000 examples have been calculated in an attempt to cover a wide parameter range systematically. This paper presents a preliminary analysis of the results. Although the accurary requirement has been relaxed somewhat with respect to 'exact' integrations in order to reach this goal, we first establish empirically that the adopted procedure does not invalidate the statistical results.

\section{Initial Conditions and Numerical Method}

The parameters of the initial system are specified as follows (Figure 1): A binary lies in the plane $A$ with its major axis in the $x$-direction and its centre of mass at $C$. Only one of the binary orbits is shown. The mean anomaly of the binary is chosen randomly within the range $[0,2 \pi]$, while its eccentricity $e$ is either zero or $e^{2}$ has a uniform random distribution in $[0,1]$. A third particle approaches in the plane $B$ with inclination $i$ and longitude of the ascending node $\Omega$. The latter is chosen randomly while the inclination is either zero (denoted a disk system) or $\cos i$ is randomized 
over its entire range. The longitude of pericentre $\omega$ is also randomized, and in most cases the semilatus rectum $s$ has a uniform random distribution between specified limits. The semimajor axis $a_{3}$ of the third particle is always such that the total energy $E<0$. The inverse of $a_{3}$ is randomized in many examples; in others $a_{3}$ is constant. There are also approximately 4000 examples where the third particle is initially close

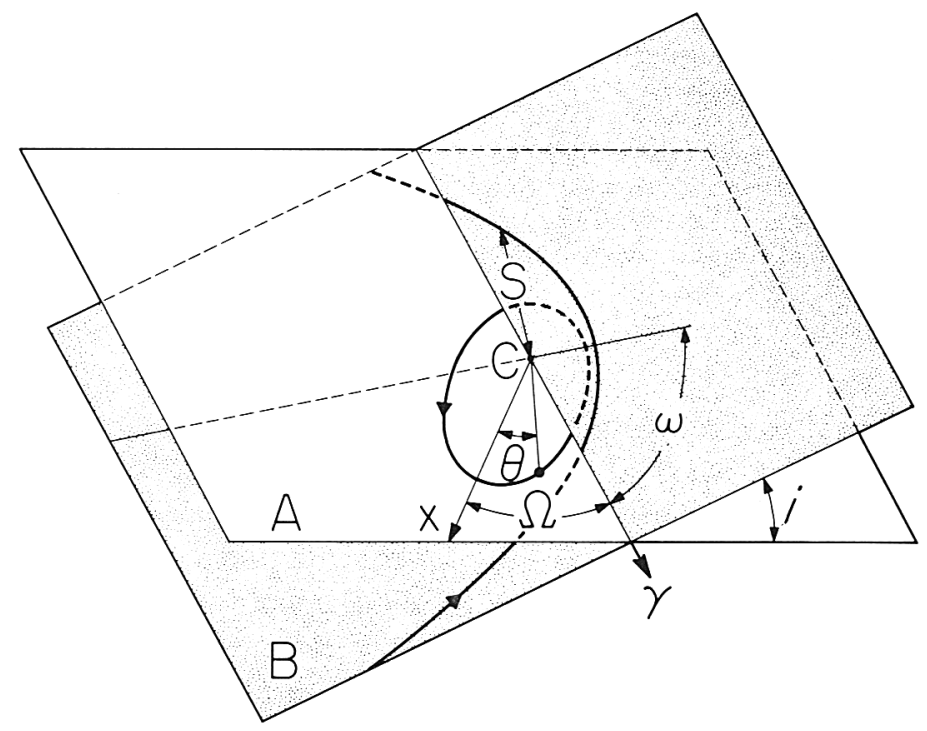

Fig. 1. Geometry of the problem. Symbols are described in the text.

to the binary and thus the orbital elements refer only to the instantaneous situation at the outset of integration. The masses denoted by $m_{1}, m_{2}, m_{3}$ have either fixed values or are chosen from a uniform random distribution within $[0.1,1]$ and normalized to make the total mass equal to unity. The semimajor axis of the initial binary is also taken to be unity. The system of units is then defined by taking the gravitational constant equal to unity.

The integration is usually started with the third particle at a distance of 20 units from the binary, unless its unperturbed orbit restricts it to shorter distances. In the latter case the integration starts with the third particle near the apocentre of its orbit. When a particle reaches 20 units from the centre of mass of the binary formed by the other two at any later time the integration is temporarily halted. The orbital elements are determined on the assumption of unperturbed two-body motion and the sign of the semimajor axis is used to decide whether escape has occurred. If the distant particle is still bound, it is moved analytically on its unperturbed orbit back to a distance of 20 units, and the binary is advanced to the corresponding phase after which the integration resumes. This procedure was also adopted by Standish (1972). Unless escape takes place before, each experiment continues until 1000 times the original binary period has elapsed. In some strongly bound triple systems an alternative time 
limit of 100 periods was adopted. A more complete description of the initial conditions is given elsewhere (Saslaw et al., 1974).

The integration method uses a fourth-order polynomial (Aarseth, 1971) together with a two-body regularization technique (Heggie, 1973). In this way close approaches between two particles can be studied without numerical difficulties while critical triple encounters are relatively rare and hence of little significance.

For each type of system we perform a large number of experiments to obtain statistically significant results. Comparisons indicate that 200-300 combined examples give a satisfactory statistical description. Also we have repeated one set of experiments with two, three and four times the standard mean integration step, each time using a different set of random numbers, to investigate the effect of integration accuracy on the final distributions.

Table I shows the rms relative energy errors, $\Delta E / E$, excluding experiments with unperturbed two-body motion. The distributions in Tables II-IV show no clear trend

TABLE I

Summary of data for accuracy tests

\begin{tabular}{llll}
\hline Set & $\left\langle(\Delta E / E)^{2}\right\rangle^{1 / 2}$ & $\begin{array}{l}\text { Number of } \\
\text { experiments }\end{array}$ & $\begin{array}{l}\text { Completed } \\
\text { (in per cent) }\end{array}$ \\
\hline I & $5 \times 10^{-4}$ & 173 & 95 \\
II & $6 \times 10^{-4}$ & 179 & 96 \\
III & $1 \times 10^{-2}$ & 162 & 97 \\
IV & $3 \times 10^{-2}$ & 208 & 96 \\
\hline
\end{tabular}

TABLE II

Eccentricity distribution (in per cent)

\begin{tabular}{lrllll}
\hline Set & $0-0.2$ & $0.2-0.4$ & $0.4-0.6$ & $0.6-0.8$ & $0.8-1.0$ \\
\hline I & 7 & 22 & 22 & 29 & 20 \\
II & 12 & 20 & 22 & 26 & 20 \\
III & 5 & 26 & 20 & 25 & 24 \\
IV & 11 & 15 & 25 & 24 & 26 \\
\hline
\end{tabular}

when going from the highest to the lowest accuracy. We are therefore confident that the accuracy of set $I$ is adequate for our investigation which also includes more difficult examples and this accuracy is used throughout. A more detailed discussion of the distributions shown in Tables II-IV is given below.

\section{General Properties}

When the third particle approaches on a nearly parabolic orbit one of three events may occur: (1) Capture, if no particle escapes within one original binary period after 
TABLE III

Terminal velocity distribution (in per cent)

\begin{tabular}{lllllrr}
\hline Set & $0-0.2$ & $0.2-0.4$ & $0.4-0.6$ & $0.6-0.8$ & $0.8-1.0$ & $>1.0$ \\
\hline I & 12 & 27 & 22 & 18 & 12 & 9 \\
II & 14 & 29 & 26 & 16 & 5 & 10 \\
III & 13 & 27 & 29 & 17 & 9 & 4 \\
IV & 11 & 26 & 28 & 19 & 10 & 7 \\
\hline
\end{tabular}

TABLE IV

Lifetime distribution (in per cent)

\begin{tabular}{lllllllll}
\hline Set & $0-2$ & $2-4$ & $4-6$ & $6-10$ & $10-20$ & $20-30$ & $>30$ \\
\hline I & 14 & 18 & 15 & 17 & 20 & 10 & 6 \\
II & 13 & 23 & 16 & 17 & 18 & 10 & 4 \\
III & 12 & 25 & 15 & 19 & 17 & 6 & 6 \\
IV & 16 & 21 & 18 & 15 & 20 & 8 & 3 \\
\hline
\end{tabular}

the first strong interaction. If a capture does not occur one has either (2) fly-by, if the third particle escapes or (3) exchange, if one of the binary particles escapes. We have not considered systems with weak interactions only, i.e. systems where the third particle having initially an elliptic orbit is still found in a similar orbit after $2000 \pi$ time units. The relative frequency of the events shows a complicated parameter dependence, but the pericentre distance $q$ of the third particle in its initial unperturbed orbit around the centre of mass of the binary is most important. In Figure 2 we show the probabili-

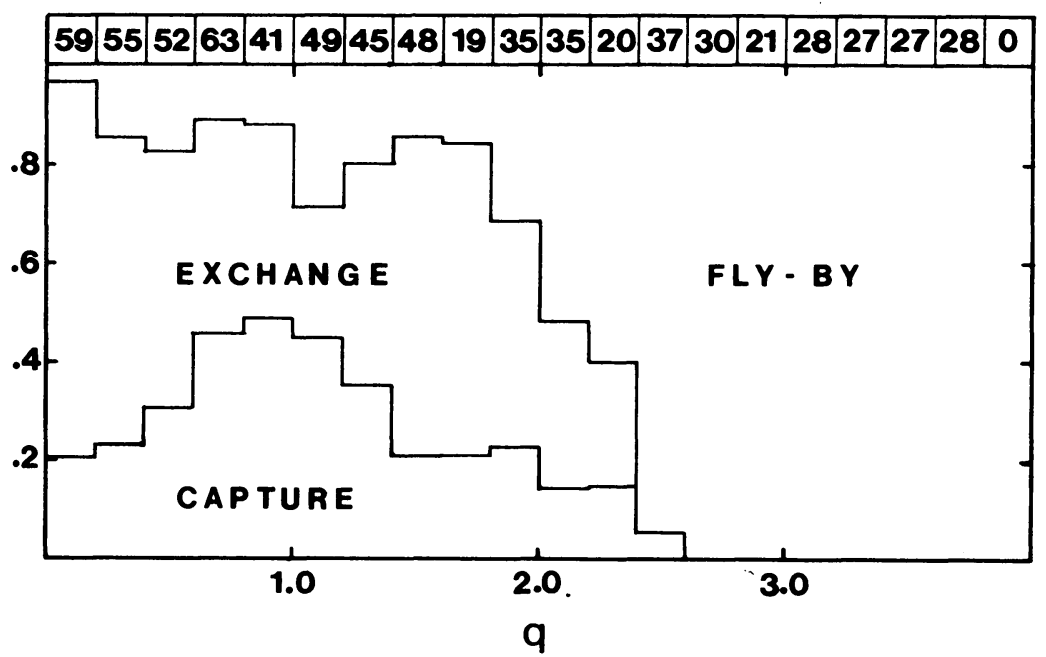

Fig. 2. Probability of the three types of motion for nearly parabolic encounters. Dẹtails are given in the text. 
ties of each event by the area occupied, as a function of the pericentre distance. The numbers on top indicate the sample size. For this set $m_{1}=m_{2}=0.25, m_{3}=0.5, a_{3}=300$, $e=0$ and $\cos i$ is randomized. If the third particle mass is decreased, the probability of exchange diminishes and disappears almost completely when $m_{3}$ is the smallest of the three particles.

When a triple system breaks up the lightest of the three particles tends to be ejected, confirming previous investigations. Figure 3 shows the frequency of escape for a

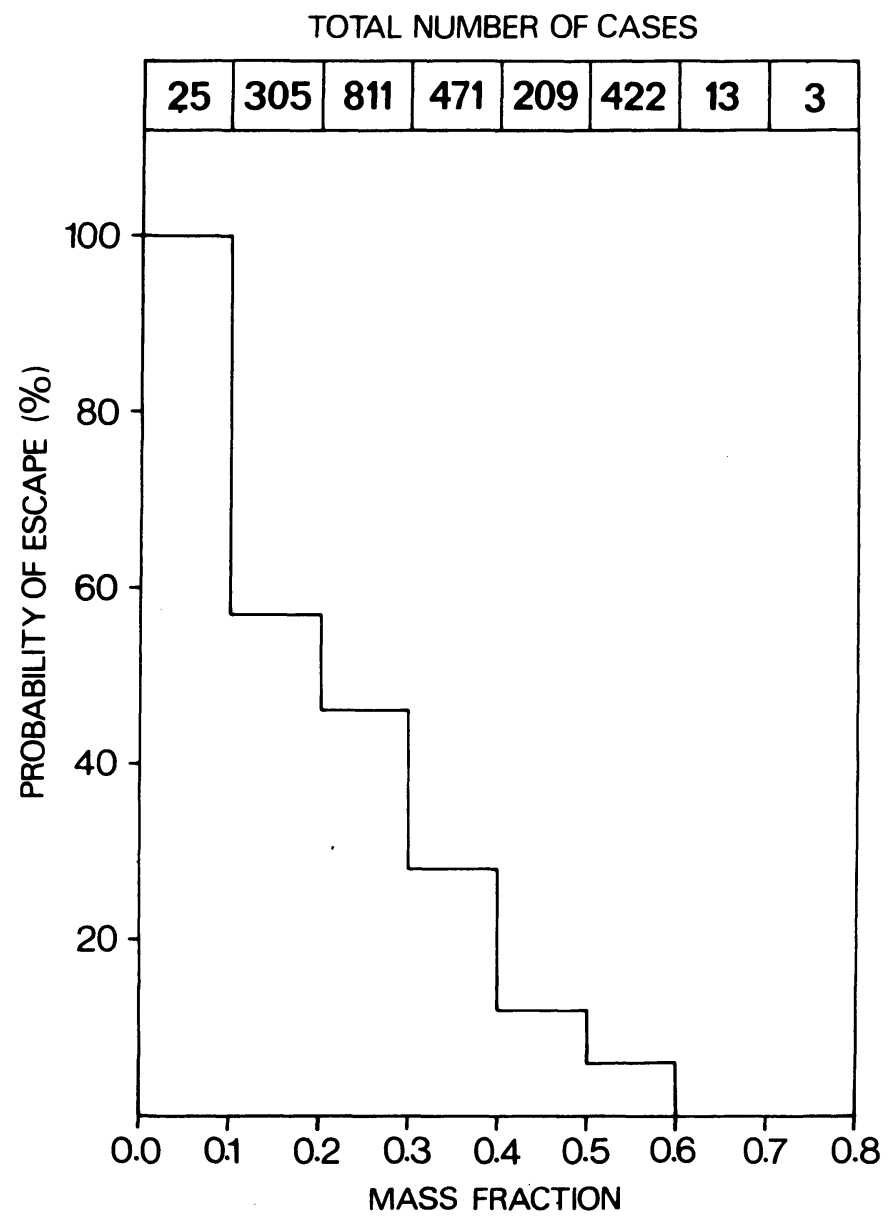

Fig. 3. Probability of escape as a function of mass.

particle of a given mass in 'both initially strongly bound systems and those created through a capture. The numbers on top again indicate the corresponding sample size. The escape probability depends on the adopted mass distribution but is not very sensitive to other parameters. It should be noted that there is a small but nonzero probability for a particle with more than half the total mass to escape. Figure 4 shows 
the probability of the incoming particle of mass 0.5 also being ejected after the interaction, as a function of impact parameter $s$ (solid line). The binary masses are both 0.25 and $e^{2}$ is random within $[0,1]$ in $\frac{3}{4}$ of all experiments and $e=0$ in the remaining $\frac{1}{4}$. The two other lines show the probability of escape for a binary component of mass $m_{2}=\frac{2}{7}$, the other particles having $m_{1}=\frac{4}{7}$ and $m_{3}=\frac{1}{7}$. The dashed line refers to a set with $e=0$ (also used for Table I), while the dotted line corresponds to $e^{2}$ randomized

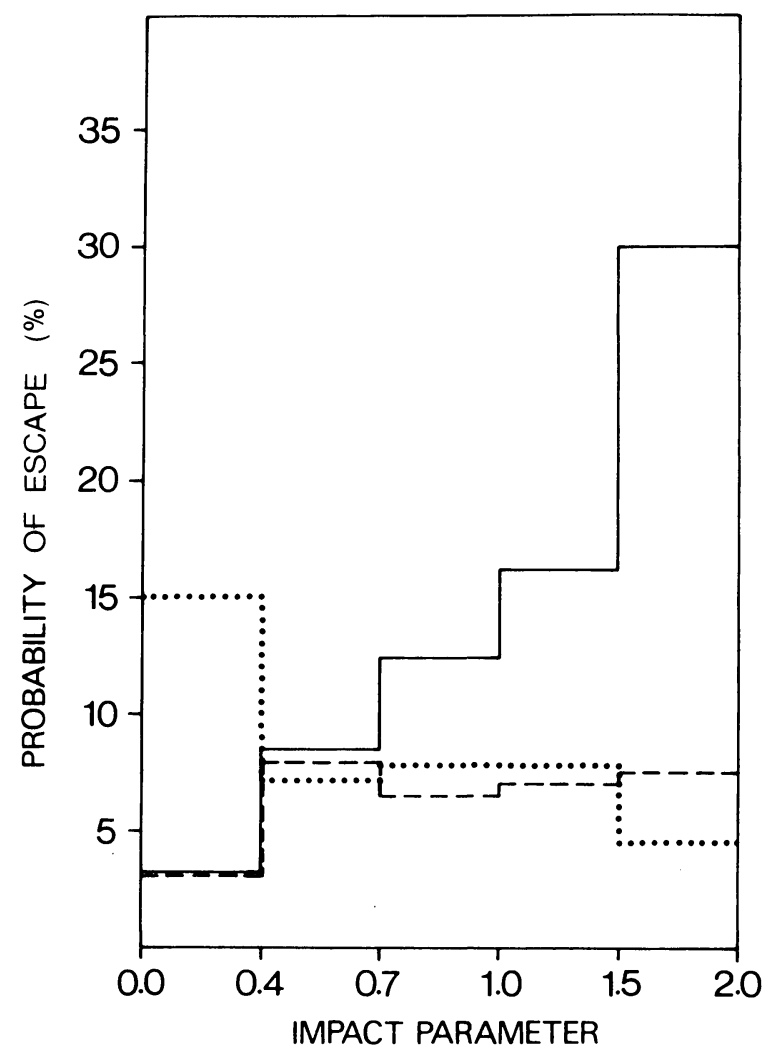

Fig. 4. Escape probability (in per cent) for a particle of given mass. The systems are specified in the text. Note that $q$ of Figure 2 is approximately equal to $0.5 s$.

in $\left[\frac{2}{3}, 1\right]$. On the other hand, the particle of mass $\frac{4}{7}$ hardly ever escapes. In all these sets $a_{3}=30$ and $\cos i$ is random in $[-1,1]$. Thus the probability of escape for a given mass also depends on its initial role. The direction in which a particle is ejected during the breakup of a triple system is not always random. Figure 5 shows the distribution of the polar angle $\theta$ of the escaped particle, measured from the direction of the rotation axis of the initial binary, for a system with $a_{3}=30, m_{1}=\frac{4}{7}, m_{2}=\frac{2}{7}, m_{3}=\frac{1}{7}, e=0$ and cos $i$ random in $\left[\frac{1}{3}, 1\right]$. Similar distributions for cos $i$ randomized in $\left[-1,-\frac{1}{3}\right]$ show a random distribution of $\cos \theta$, and for $\cos i$ randomized in $\left[-\frac{1}{3}, \frac{1}{3}\right]$ one has a slight preference for the binary plane $\theta=90^{\circ}$. This trend becomes more pronounced for low 
inclination systems. We have also measured the polar angle of escape from the direction of the total angular momentum of the triple system, as illustrated in Figures 6 and 7 on the right-hand side. On the left we show the distribution of polar angles of the incoming directions of the third particle. The set of experiments included in Figures 6 and 7 have cosi randomized within $\left[\frac{1}{3}, 1\right]$ and $\left[-1,-\frac{1}{3}\right]$, respectively. The final distributions are more concentrated towards the plane normal to the total angular

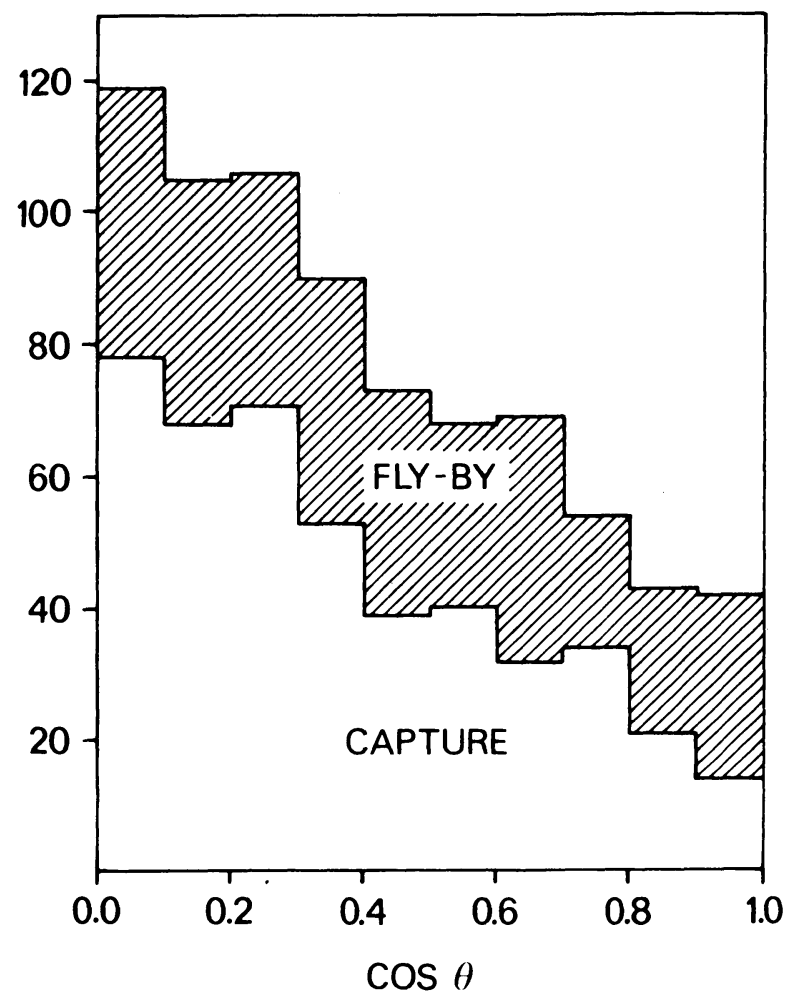

Fig. 5. Number distribution of the polar angle for the escaping particle. The angle $\theta$ is measured with respect to the initial rotation axis of the binary.

momentum than the initial ones. This effect disappears, however, when the third particle mass is increased to values comparable with the binary components. In all three previous figures the fly-by examples are shown as shaded areas to distinguish them from captures. No exchanges occurred in this set.

The escape energy is strongly peaked near zero as shown in Figure 8. The data are obtained from the same set as that of the three previous figures, with cosi random in $[-1,1]$ and $e=0$ (solid line). The dashed line refers to a similar set except $e^{2}$ is random in $\left[\frac{2}{3}, 1\right]$. The escape energy appears more likely to have a large value in the latter set. In general the escape energy is negatively correlated with the minimum of the moment of inertia relative to the centre of mass. 


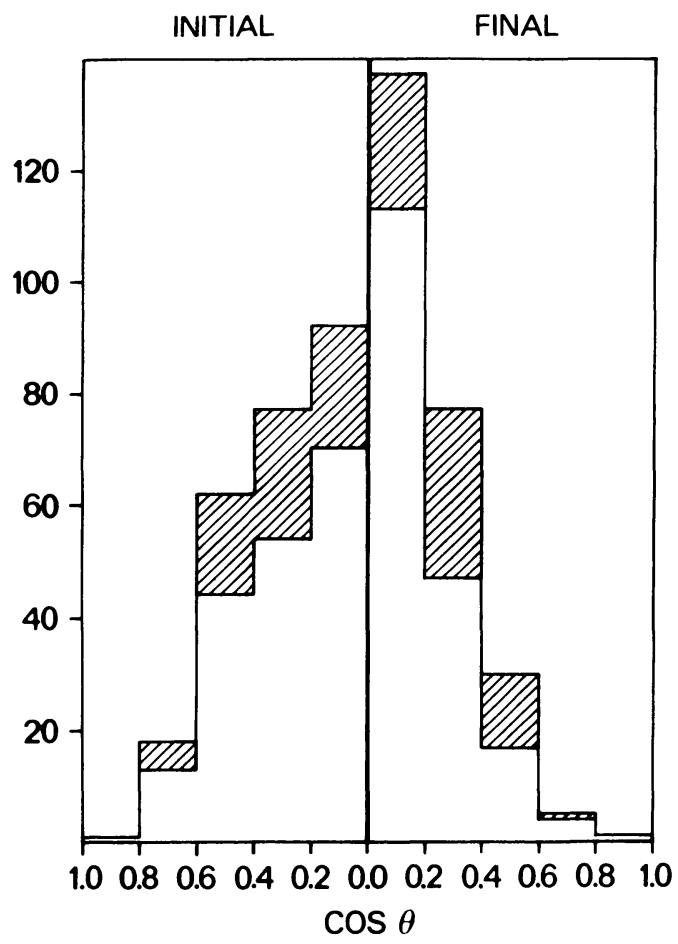

Fig. 6. Number distribution of the polar angle for the incoming (left) and escaping (right) particle. The angle $\theta$ is measured with respect to the direction of total angular momentum. Initial inclinations are in the range $0^{\circ}-70^{\circ} .5$. Further details are given in the text.

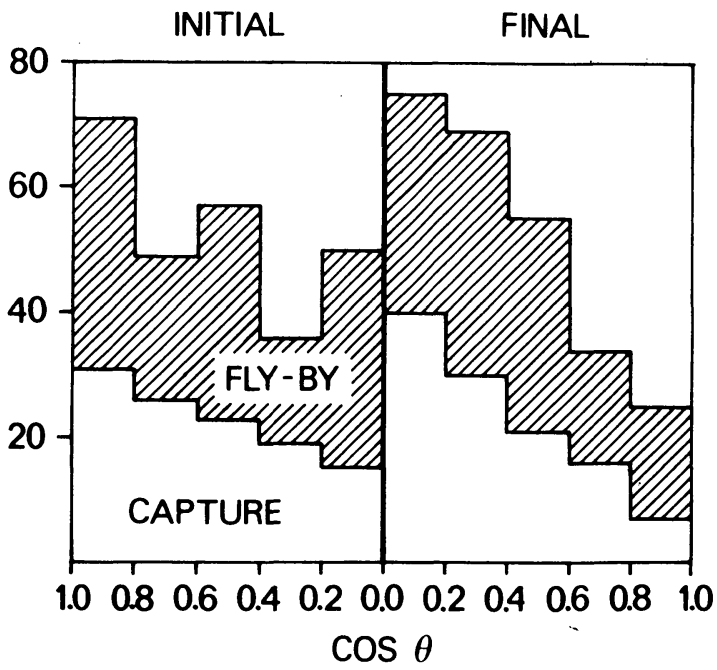

Fig. 7. Number distribution of the polar angle for the incoming (left) and escaping (right) particle. The angle $\theta$ is measured with respect to the direction of total angular momentum. Initial inclinations are in the range $109^{\circ} .5-180^{\circ}$. Further details are given in the text. 


\section{Close Triples and Captures}

Initially strongly bound systems and triples formed by captures are found to behave similarly if the main parameters are similar, and therefore they can be studied together. Here we concentrate on the final distributions of the eccentricity of the binary which is left behind after an escape, the distribution of the relative velocity (at infinity) of the escaping particle, and the distribution of lifetimes of triple systems measured in cros-

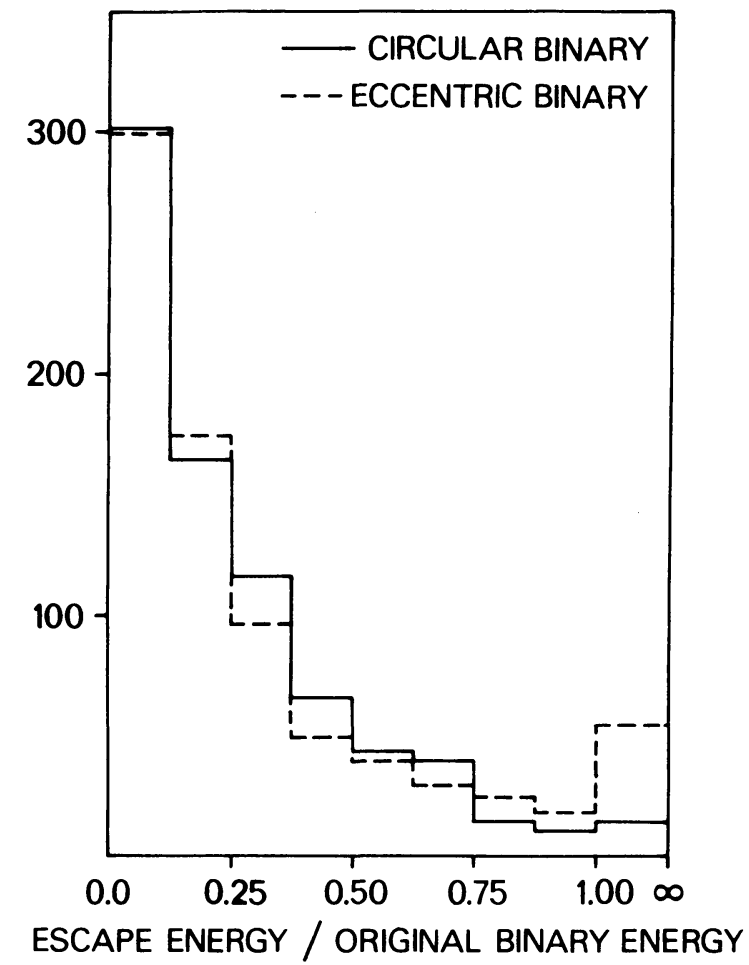

Fig. 8. Number distribution of escape energy in terms of the original binary energy. Details are given in the text.

sing times defined by $T_{\mathrm{cr}} \equiv|2 E|^{-3 / 2}$ in our units. Results are shown in Table $\mathrm{V}$ for a few sets chosen to demonstrate the effect of various parameters. The columns give the number of experiments included in each set, the mean energy $\langle E\rangle$ and mean total angular momentum $\langle h\rangle$ as well as the mean mass range $\left\langle m_{\max } / m_{\min }\right\rangle$ for each set; the times (in units of the crossing time) by which one quarter, one half or three quarters of the systems are terminated $\left(T_{1 / 4}, T_{1 / 2}, T_{3 / 4}\right)$; and the mean of the terminal escape velocity $\left\langle v_{\infty}\right\rangle$.

The first six sets have $a_{3}=30$ and fixed masses in the order $m_{1}, m_{2}, m_{3}$ as indicated in the last column. In set $5, e=0$; in others $e^{2}$ is random within $[0,1]$ in $\frac{3}{4}$ of all examples and $e=0$ in the remaining $\frac{1}{4}$. The impact distance $s$ is always $\leqslant 2$, and $\cos i$ is random in $[-1,1]$. Comparison of sets $1-4$ shows that increasing the mass range makes the 
TABLE V

Summary of results

\begin{tabular}{lllllrrrrr}
\hline Set & $\begin{array}{l}\text { Number of } \\
\text { experiments }\end{array}$ & $\langle E\rangle$ & $\langle h\rangle$ & $\left\langle m_{\max } / m_{\min }\right\rangle$ & $T_{1 / 4}$ & $T_{1 / 2}$ & $T_{3 / 4}$ & $\left\langle v_{\infty}\right\rangle$ & Notes \\
\hline 1 & 327 & -0.06 & 0.22 & 1.0 & 10 & 28 & 75 & 0.42 & $1,1,1$ \\
2 & 250 & -0.05 & 0.25 & 1.5 & 8 & 22 & 54 & 0.38 & $1,1,1.5$ \\
3 & 263 & -0.04 & 0.24 & 2.0 & 4 & 15 & 45 & 0.40 & $1,2,2$ \\
4 & 217 & -0.04 & 0.25 & 2.0 & 4 & 13 & 42 & 0.37 & $1,1,2$ \\
5 & 483 & -0.08 & 0.21 & 4.0 & 9 & 26 & 68 & 0.48 & $4,2,1$ \\
6 & 219 & -0.12 & 0.19 & 20.0 & 4 & 13 & 40 & 0.63 & $20,20,1$ \\
& & & & & & & & & \\
7 & 216 & -0.16 & 0.20 & 1.3 & 15 & 41 & 92 & 0.55 & \\
8 & 377 & -0.12 & 0.22 & 2.5 & 8 & 22 & 57 & 0.54 & \\
9 & 340 & -0.09 & 0.23 & 5.7 & 4 & 10 & 32 & 0.53 & \\
& & & & & & & & & \\
10 & 240 & -0.09 & 0.03 & 3.3 & 4 & 9 & 20 & 0.66 & \\
11 & 469 & -0.14 & 0.14 & 2.8 & 5 & 13 & 40 & 0.70 & \\
12 & 503 & -0.13 & 0.25 & 2.8 & 7 & 19 & 47 & 0.51 & \\
13 & 240 & -0.09 & 0.27 & 3.2 & 6 & 18 & 48 & 0.47 & \\
14 & 441 & -0.10 & 0.36 & 2.7 & 10 & 30 & 100 & 0.36 & \\
& & & & & & & & & \\
15 & 374 & -0.02 & 0.32 & 2.9 & 4 & 11 & 40 & 0.36 & Random \\
16 & 118 & -0.02 & 0.33 & 3.2 & 3 & 8 & 20 & 0.37 & Disk \\
17 & 139 & -0.02 & 0.42 & 2.7 & 4 & 8 & 30 & 0.32 & Disk \\
& & & & & & & & & \\
18 & 120 & -0.17 & 0.29 & 1.0 & 10 & 40 & 150 & 0.52 & Disk \\
\hline
\end{tabular}

systems less stable while the escape velocity is not affected. Comparison of sets 3 and 4 indicates that the results are relatively insensitive to the intermediate mass. Sets 7-9 which contain a range of masses initially strongly bound show the same phenomenon. By comparing sets 6 and 8 we note that the instability increases with mass range even when the latter is large already. Furthermore, the escape velocity increases in this case.

Sets 10-14 demonstrate the effect of the total angular momentum $h$. These are also strongly bound systems with a range of masses. The stability increases with angular momentum while the escape velocity behaves in the opposite way. Comparison of sets $15-17$ or 7 and 18 shows that the third dimension is not important from the point of view of stability or escape velocity. However, because of the small sample size it is not possible to rule out a small effect. In sets 15-17 the parameters are: $m_{1}=m_{2}=1$, $m_{3}$ is random in $[1.2,2.8],-400<a_{3}<-6.7, s<3$ and $e^{2}$ is random within $[0,1]$ in $\frac{3}{4}$ of all examples and $e=0$ in the remaining $\frac{1}{4}$. Set 15 has random orientations and sets 16 and 17 are disk systems. Finally set 18 consists of equal masses initially strongly bound in a disk.

Comparing sets 12-14 with 15-17 we see that the strongly bound systems are more stable and produce higher escape velocities than systems created by capture. The same effect can be seen by comparing sets 1 and 2 with 7 or sets 3 and 4 with 8 . Figure 9 shows the actual distributions of the escape velocity and eccentricity for sets $10,11,13$ 
and 14. These results from the low angular momentum set $10(h=0.027)$ may be compared with earlier results of zero angular momentum systems (e.g. Standish, 1972; Szebehely, 1972). Although the initial conditions are slightly different, the final distributions of lifetime, eccentricity and velocity are in good agreement. Other similar calculations (Agekyan and Anosova, 1968; Anosova, 1969a, b) employ different escape criteria and cannot be compared directly with our results. However, the lifetime dependence on mass range and angular momentum is still in qualitative agree-
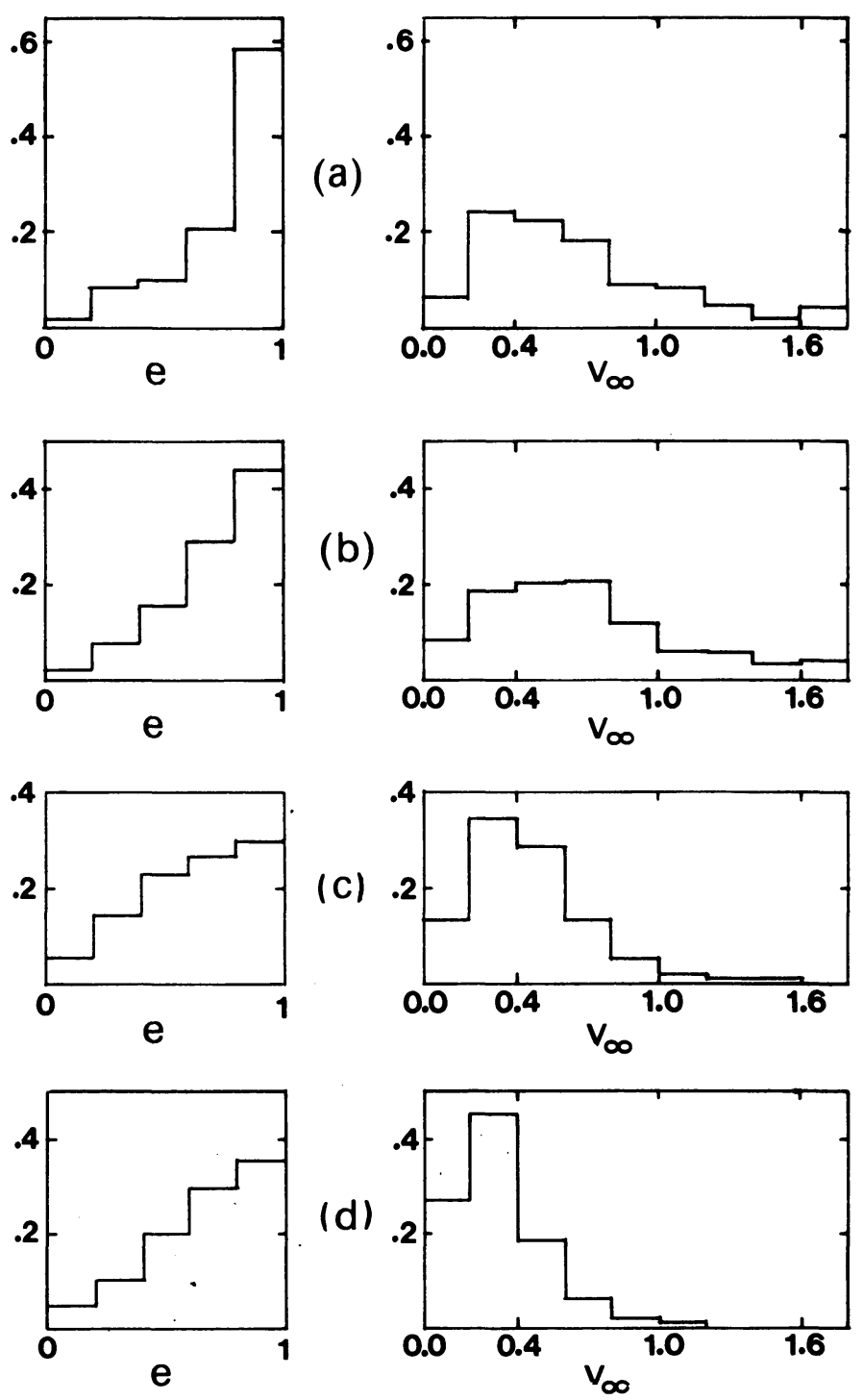

Fig. 9. Distributions of final eccentricity and escape velocity for different angular momenta. (a) $h=0.027$; (b) $\langle h\rangle=0.14$; (c) $h=0.27$; (d) $\langle h\rangle=0.36$. The results refer to sets $10,11,13,14$ of Table V. 
ment. The effect of the main parameters is summarized in Figure 10. The arrows point in the direction of increase for each of the quantities, and the absence of an arrow implies approximate constancy over the corresponding range of parameter values. For example, the eccentricity does not vary appreciably when the angular momentum changes from 0.2 to 0.4 but decreases when $h$ increases from 0.03 to 0.2 . Question marks indicate a constancy which is not well established.

\begin{tabular}{|c|c|c|c|}
\hline Parameter & $\mathbf{T}$ & $\mathbf{v}_{\infty}$ & e \\
\hline $\begin{array}{r}0.03 \\
0.2 \\
0.4\end{array}$ & $\downarrow$ & $\uparrow$ & $\uparrow$ \\
\hline $\begin{array}{r}-0.15 \\
-0.02\end{array}$ & $\uparrow$ & $\uparrow$ & $?$ \\
\hline $\mathbf{m}_{\max } \quad \begin{array}{r}5 \\
\mathbf{m}_{\min } 20\end{array}$ & $\uparrow$ & & $\uparrow$ \\
\hline i Disk \\
Random
\end{tabular}

Fig. 10. Qualitative dependence of lifetime, escape velocity and final eccentricity on the main parameters. Arrows point in the direction of increase as explained in the text.

After a preliminary analysis of the experiments it appears that strongly bound threebody systems and those created by capture can be described by only a few parameters, the most important of which are the total angular momentum (Standish, 1972), the total energy and the mass range (Heggie, 1972). Furthermore, a relatively small sample is sufficient to obtain statistically significant distributions. Comparisons of three- and two-dimensional systems have shown their similarity and therefore support the general validity of the results obtained before. A more extensive analysis is to be undertaken to study the significance of other parameters.

\section{Acknowledgements}

It is a pleasure to record the constant help and encouragement in this project from my supervisor, Dr S. J. Aarseth. I also wish to thank Dr W. C. Saslaw and Dr D. C. Heggie for assistance and helpful discussions. The author is supported by an Osk. Huttusen Säätiö Foundation Fellowship. 


\section{References}

Aarseth, S. J.: 1971, Astrophys. Space Sci. 14, 118.

Agekyan, T. A. and Anosova, Zh. P.: 1967, Soviet Astron. 11, 1006.

Agekyan, T. A. and Anosova, Zh. P.: 1968, Astrophys. 4, 11.

Anosova, Zh. P.: 1969a, Astrophys. 5, 81.

Anosova, Zh. P.: 1969b, Publ. Astron. Obs. Leningrad 26, 88.

Heggie, D. C. : 1972, Thesis, Cambridge University.

Heggie, D. C.: 1973, in B. D. Tapley and V. Szebehely (eds.), Recent Advances in Dynamical Astronomy,

D. Reidel, Dordrecht, The Netherlands, p. 34.

Saslaw, W. C., Valtonen, M. J., and Aarseth, S. J.: 1974, Astrophys. J. (to be published).

Standish, E. M.: 1972, Astron. Astrophys. 21, 185.

Szebehely, V.: 1972, Celes. Mech. 6, 84.

Szebehely, V. and Peters, C. F.: 1967, Astron. J. 72, 876.

\section{DISCUSSION}

R. H. Miller: How do your results compare with earlier works by Yabushita and others?

$M$. J. Valtonen: The work of Agekyan and Anosova was mainly concerned with planar systems and usually equal or fixed masses. The same applies to the work by Standish and Szebehely, while Yabushita was concerned with systems of positive total energy. Our results agree with the previous works as far as we have been able to check.

$P$. Stewart: The experiments were originally conceived for the purpose of explaining why radio sources have double structure. Do you have any thought on the more complex structure involving sometimes four radio components on each side of an optical galaxy?

$M$. J. Valtonen: In the case of radio-source models one is not restricted to three-body systems alone, and in fact models with four or more bodies have also been studied. 